\title{
The H2020 VERTIGO project towards tbit/s optical feeder links
}

\author{
A. Le Kernec, L. Canuet, A. Maho, M. Sotom, D. Matter, et \\ al.
}

A. Le Kernec, L. Canuet, A. Maho, M. Sotom, D. Matter, L. Francou, J. Edmunds, M. Welch, E. Kehayas, N. Perlot, M. Krzyzek, Anagnostis Paraskevopoulos, J. Leuthold, Y. Horst, J. Bourderionnet, A. Brignon, E. Lallier, V. Billault, L. Leviandier, J.-M. Conan, N. Védrenne, C. B. Lim, A. Montmerle-Bonnefois, C. Petit, L. Stampoulidis, M. Fehrenz, T. LehnigkEmden, "The H2020 VERTIGO project towards tbit/s optical feeder links," Proc. SPIE 11852, International Conference on Space Optics - ICSO 2020, 1185217 (11 June 2021); doi: 10.1117/12.2599229

SPIE Event: International Conference on Space Optics - ICSO 2021, 2021, Online Only 


\section{International Conference on Space Optics-ICSO 2020}

Virtual Conference

30 March-2 April 2021

Edited by Bruno Cugny, Zoran Sodnik, and Nikos Karafolas
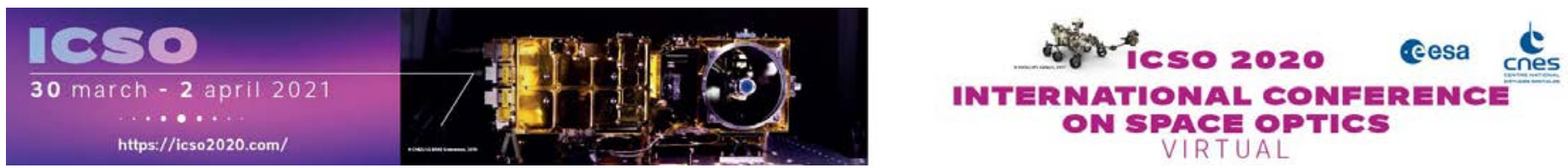

\section{The H2020 VERTIGO project towards tbit/s optical feeder links}

\section{Cesa isso procedings denes}




\title{
The H2020 VERTIGO Project Towards Tbit/s Optical Feeder Links
}

\author{
A. Le Kernec*a, L. Canuet ${ }^{\mathrm{a}}$, A. Maho ${ }^{\mathrm{a}}$, M. Sotom ${ }^{\mathrm{a}}$, D. Matter ${ }^{\mathrm{b}}$, L. Francou ${ }^{\mathrm{b}}$, J. Edmunds ${ }^{\mathrm{c}}, \mathrm{M}$. \\ Welch $^{\mathrm{c}}$, E. Kehayas ${ }^{\mathrm{c}}$, N. Perlot ${ }^{\mathrm{d}}$, M. Krzyzek ${ }^{\mathrm{d}}$, Anagnostis Paraskevopoulos ${ }^{\mathrm{d}}$, J. Leuthold ${ }^{\mathrm{e}}, \mathrm{Y}$. \\ Horst $^{\mathrm{e}}$, J. Bourderionnet ${ }^{\mathrm{f}}$, A. Brignon ${ }^{\mathrm{f}}$, E. Lallier ${ }^{\mathrm{f}}$, V. Billault ${ }^{\mathrm{f}}$, L. Leviandier ${ }^{\mathrm{f}}$, J.-M.. Conan $^{\mathrm{g}}$, N. \\ Védrenne $^{\mathrm{g}}$, C. B. Lim $^{\mathrm{g}}$, A. Montmerle-Bonnefois ${ }^{\mathrm{g}}$, C. Petit ${ }^{\mathrm{g}}$, L. Stampoulidis ${ }^{\mathrm{h}}$, M. Fehrenz ${ }^{\mathrm{i}}, \mathrm{T}$. \\ Lehnigk-Emden ${ }^{\mathrm{i}}$
}

${ }^{a}$ Thales Alenia Space in France, 26 Avenue J.F. Champollion - B.P. 33787, 31037 Toulouse Cedex 1, France;

${ }^{\mathrm{b}}$ Thales Alenia Space in Switzerland, Schaffhauserstrasse 580, Zürich CH-8052, Switzerland.

${ }^{\mathrm{c}} \mathrm{G} \& \mathrm{H}$ Torquay, Broomhill Way, Torquay, TQ2 7QL, United Kingdom

${ }^{\mathrm{d}}$ Fraunhofer Heinrich-Hertz Institute, Einsteinufer 37, 10587 Berlin, Germany

${ }^{\mathrm{e}}$ Institute of Electromagnetic Fields (IEF), ETH Zurich, Gloriastrasse 35, 8092 Zurich, Switzerland

${ }^{\mathrm{f}}$ Thales Research \& Technology, 1 Avenue Augustin Fresnel, 91767 Palaiseau Cedex, France

${ }^{g}$ ONERA, DOTA, Paris Saclay University, F-92322 Châtillon, France

${ }^{h}$ LEO Space Photonics R\&D, Lefkippos Tech. Park, 27 Neapoleos Str., Ag. Paraskevi, 15341,

Athens, Greece

${ }^{i}$ Creonic GmbH, Bahnhofstrasse 26-28, 67655 Kaiserslautern, Germany

\begin{abstract}
To concurrently cope with the scarcity of RF frequency bands, the growing capacity demand and the required lower cost of the ground segment, Very High Throughput Satellites systems must rely on new technical solutions. Optical feeder links are considered as a promising alternative to surpass classical RF technology, offering assets inherent to optical technologies (large bandwidth, no frequency regulation, low beam divergence, components availability). Nevertheless the potential of this technology shall not conceal the remaining challenges to be overcome to make it relevant for operational missions: clouds, turbulence, power generation and high efficiency modulations.

VERTIGO (Very High Throughput Satellite Ground Optical Link) is a 3-year H2020 project funded by the European commission and started mid-2019 focusing on the optical link itself regardless of site diversity aspect and aiming at demonstrating in ground demonstrations required technologies to implement very high capacity optical feeder links. In this paper we present the current status and perspectives of the project.
\end{abstract}

Keywords: optical feeder links, high throughput, waveform, high optical power, atmospheric turbulence, mitigation techniques

\section{INTRODUCTION}

During the past decade, telecom satellite systems have experienced a significant capacity increase to cope with the advent of new applications and usage and have achieved capacities in the Tbit/s capacities for a single GEO satellite. Specifically, the capacity of such communication systems increased from roughly $100 \mathrm{Gbit} / \mathrm{s}$ at the beginning of the 2010's (Ka-sat, KONNECT, Jupiter-1, Viasat-1) to several 100's Gbit/s and up 1 Tbit/s at beginning of the 2020's (Jupiter 3-EchoStar 24, coming KONNECT VHTS, coming Viasat-3 satellites). In order to feed such very high capacity satellites, a large number of RF ground stations are necessary (several tens) monopolizing RF bands over a large area while generating positioning constraints and stringent RF requirements to other satellites in order to avoid interferences. Optical feeder links are a promising alternative to classical RF counterparts offering high throughput transmission capabilities while freeing RF spectra, avoiding interference issues and possibly reducing the ground segment. An overview of the different optical feeder link architecture options were described for example in [1], [2] and [3].

*arnaud.le-kernec@thalesaleniaspace.com 
Despite the huge potential of this technology, technical and operational challenges need to be overcome in order to confirm the viability of this solution, including but not limited to:

- Availability of the feeder link vs. number and locations of the optical ground stations

- Highly efficient (spectrally and in performance) modulation schemes

- High and very high optical power generation and management

- High power multiplexing

- Atmospheric impairments mitigation (through optical and digital means)

The H2020 VERTIGO project focuses on the optical link technology gaps regardless of cloud coverage issues.

\section{METHODOLOGY}

\subsection{The key technologies}

The ambition of VERTIGO is to implement and test in lab and outdoor ground-ground demonstrations technologies required for the development of high capacity satellite systems based on optical feeder links. To this aim a dedicated consortium has been built, gathering necessary competences to address the technical and technological challenges, composed of: Creonic GmbH, ETHZ, Fraunhofer HHI, Gooch \& Housego, Leo Space Photonics, ONERA, Thales Alenia Space, Thales Research \& Technology [4].

The following key technologies are covered:

- Efficient modulation formats: digital modulation schemes including both IM/DD and coherent detection as well as RF analog modulation will be tested.

- High optical power generation: two classes of optical amplifiers, $10 \mathrm{~W}$ and $50 \mathrm{~W}$, will be developed and tested. To achieve upper-level optical power in the $100 \mathrm{~W}$ class, coherent power combining will be implemented and verified experimentally.

- High power wavelength multiplexing: multiple wavelengths will be required on downlink and uplink to achieve high throughput, each requiring as a minimum multi-watt power. Incoherent combination (multiplexing) of these high power channels is thus a critical function that will be developed and tested.

- Adaptive optics and multi-aperture optics: to mitigate turbulence effects through optical techniques, two different optical ground stations techniques will be implemented based on single-aperture with adaptive optics and multi-aperture with tip-tilt correction offering the opportunity to compare both approaches.

- Error correction coding/interleaving : as a complementary technique, inseparable of optical means to mitigate the turbulence effect on signal, coding/interleaving enable the recovery of data. 


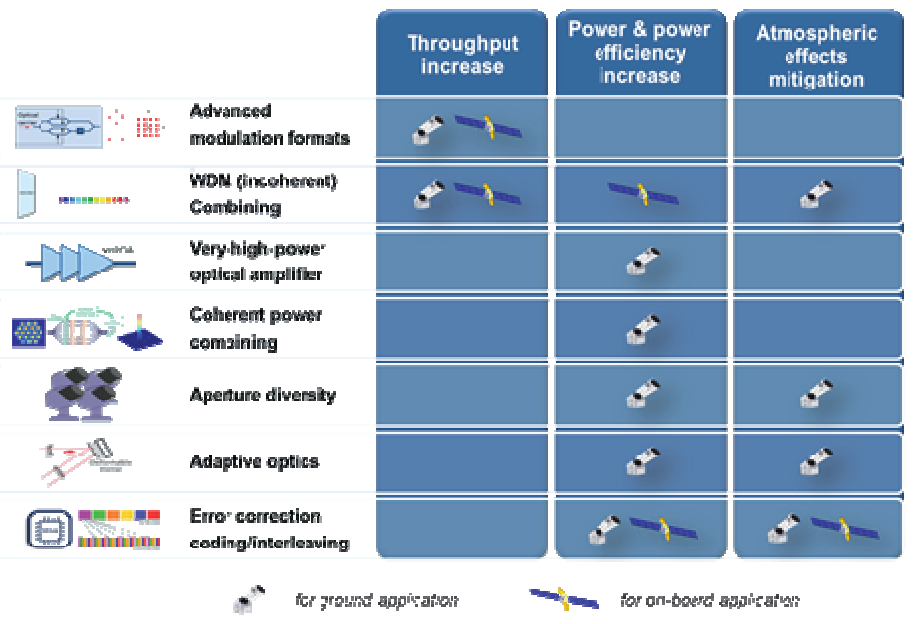

Figure 1. Key technologies developed and demonstrated in VERTIGO.

\subsection{Laboratory and outdoor system demonstrations}

Two demonstrations will take place in the frame of VERTIGO each covering a specific scope of tests. The first one will take place in a laboratory environment in Paris area and will focus on the optical telecom chain through the evaluation of the impact of high and very high optical power on the integrity of the transmitted telecom signal by integrating in a same set-up, high power generation building blocks, high power optical multiplexing, incoherent modulation schemes (digital and analog) and channel coding/interleaving.

The second demonstration will take place in Switzerland and will evaluate in representative (or worst case) conditions (53 km link through the atmosphere), and both directions (uplink and downlink), the feasibility of very high throughput feeder links, addressing the advanced concepts developed in the frame of the project: digital incoherent/coherent and analog modulation schemes, channel coding/interleaving, high power amplifiers, multi-aperture and adaptive optics in emission and reception, space terminal emulator. This state-of-the-art demonstration will give the opportunity to test and compare different techniques and technologies (modulation formats, adaptive optics and multi-aperture techniques) in a relevant environment, to consolidate theoretical models for the performance predictions, and evaluate the relevance of the different techniques for an implementation in future operational systems.

\section{INDOOR DEMONSTRATION}

\subsection{Demonstration overview}

The purpose of the indoor (in a lab environment) demonstration is twofold: first, to demonstrate specific technologies requiring a particular environment and/or which cannot be demonstrated under safe conditions outdoor, second, to validate as a first step, in a well-mastered environment the technologies to be implemented in the field demonstration. Figure 2 presents the scheme of the demonstration. It will be composed of all the building blocks involved in the communication chain: optical transmitters, optical receivers, high power amplifiers, high power multiplexer, coherent combiner, preamplifier and demultiplexer. A fibered channel emulator will be used to apply a variable attenuation representative of the turbulence effect to the optical signal (based on simulated time series). Coding/interleaving and deinterleaving/decoding stages will be applied off-line. One of the main objectives of this demonstration will be to evaluate the impact of high and very optical power transmission on telecom signal performance. 


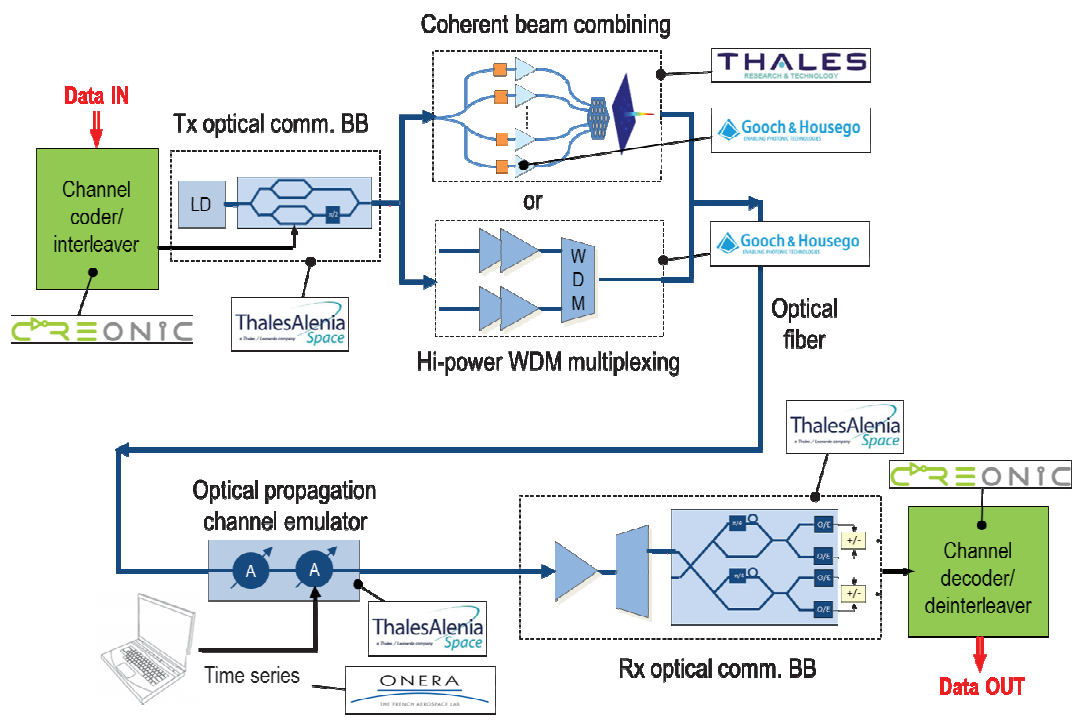

Figure 2. General scheme of the indoor demonstration.

The following sections detail the different parts of the demonstrator.

\subsection{Optical communication transmitters and receivers}

In line with the different optical feeder link architectures described in [3], several modulation formats will be implemented:

- Analog modulation chain (RF-over-FSO) implemented by Thales Alenia Space France: due to the easiness of implementation of this modulation format in telecom payload, (limited modifications required) this option is still considered as a potential candidate in spite of the lack of robustness to atmospheric impairments.

- $\quad$ NRZ-OOK and NRZ-DPSK at 25 Gbps and NRZ-DQPSK at 50 Gbps comm chains (with Direct of Differential Detection) implemented by Thales Alenia Space France

- Coherent transmission chain implemented by ETHZ: QPSK modulation will be the baseline with a symbol rate in the $25-32$ Gbaud range.

Pictures of the breadboards are presented in the figures hereafter.

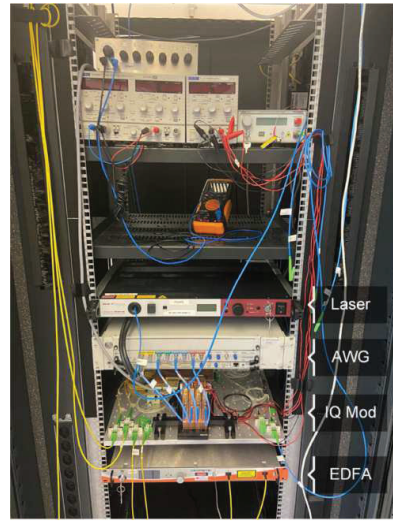

Optical Tx Breadboard/Rack

Figure 3. Coherent transmitter and receiver breadboards provided by ETHZ.

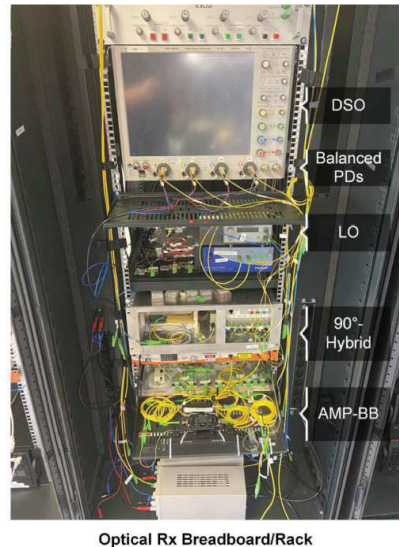

Optical Rx Breadboard/Rack

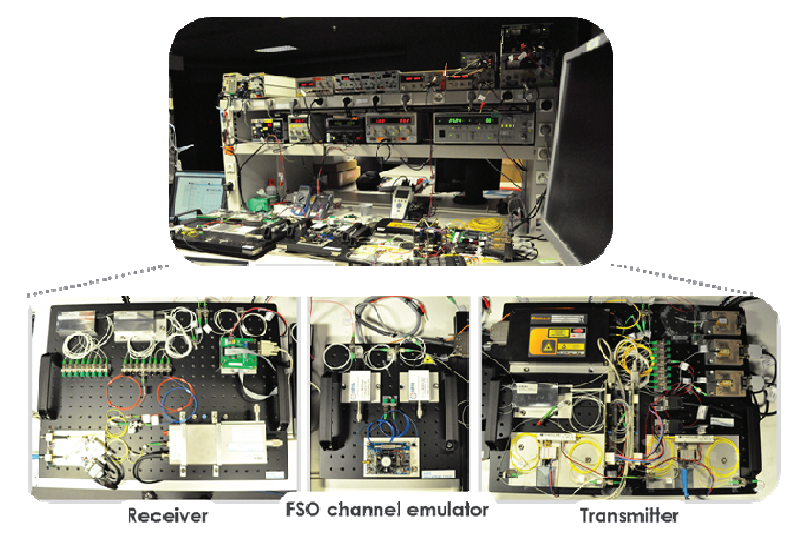

Figure 4. IM/DD transmitter and receiver breadboards provided by Thales Alenia Space France. 
The digital transmission will be protected through a specific coding/interleaving scheme developed by CREONIC and relying on concatenated RS codes and interleaver stages.

\subsection{High and Very high optical power generation}

Generation of high and very high optical power is a key enabler for optical feeder links which will be tested in laboratory environment for obvious safety reasons. Two classes of amplifiers have been designed by Gooch \& Housego and Leo Space Photonics, namely $10 \mathrm{~W}$-class amplifier and $50 \mathrm{~W}$-class amplifier, addressing downlinks and uplinks respectively. The 10W-class amplifier relies on a 3 -stage architecture while the $50 \mathrm{~W}$-class amplifier builds on the $10 \mathrm{~W}$ design with an additional power amplifier stage. Both amplifiers make use of high power multi-mode pump diodes at 9xx nm. Long wavelengths (beyond $1550 \mathrm{~nm}$ ) are assumed to be used for uplink with the highest power requirements per wavelength. Shorter wavelengths are assumed to be used for downlink. Numerical modeling was performed to simulate and predict the performance of the amplifiers. The figures below provide first assessment of the expected output power and noise figure over the C-band.

\section{W amplifier}
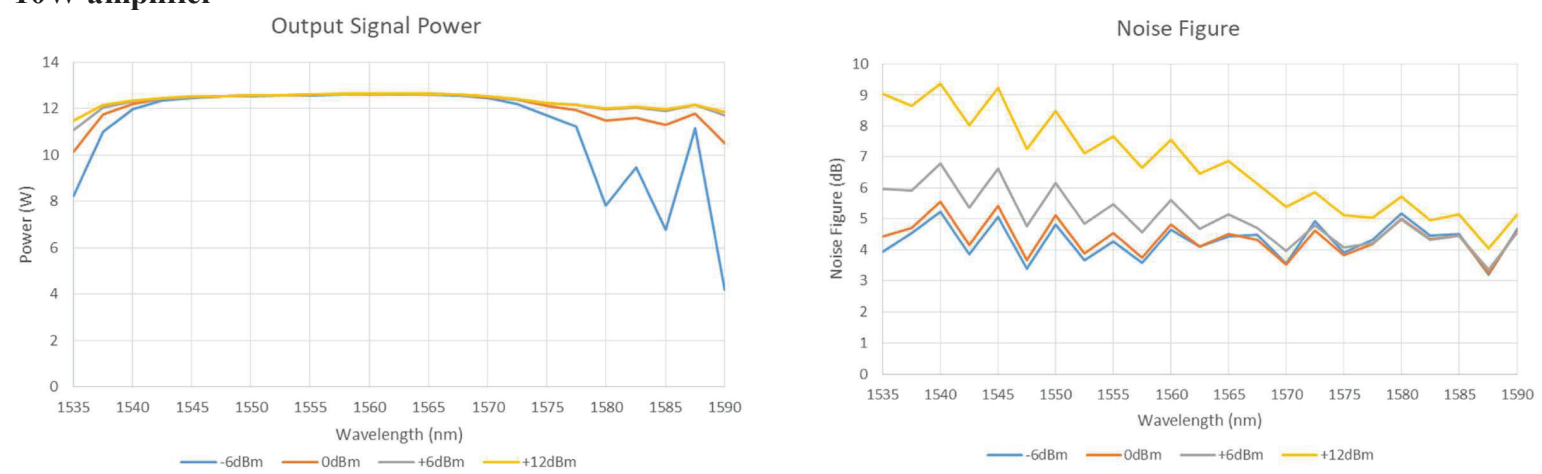

50W amplifier
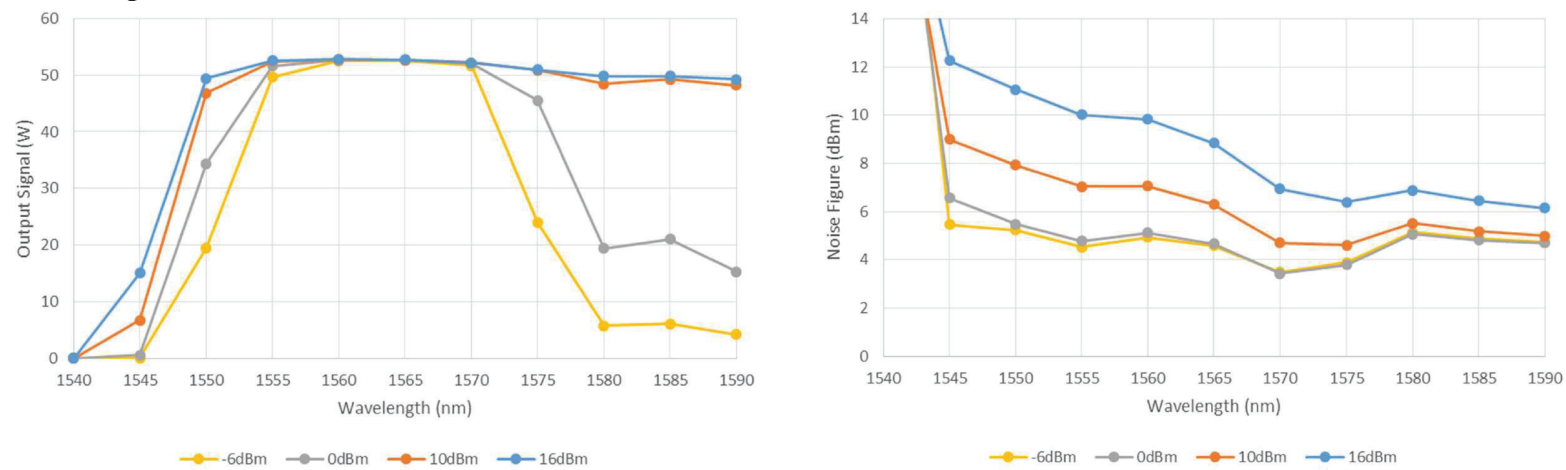

Figure 5. 10W-class and 50W-class amplifier simulated performance over wavelength (colors correspond to amplifier input power).

To reach $100 \mathrm{~W}$-class optical power, two 50W-class optical amplifier outputs will be coherently combined. The general concept of the coherent beam combination (CBC) is presented in Figure 6. The incident optical field is split in two replica by a 50/50 beam splitter to subsequently feed two amplifiers in parallel configuration. The optical beams from the two amplifiers are then recombined through a second 50/50 beams splitter. A delay line in one arm allows to phase-lock the two signals. Unlike the usual CBC application field, in VERTIGO we combine beams carrying a spectral telecom content and possibly several wavelengths. The theoretical impact of a residual delay between arms on the coupling 
efficiency in the combination of 40 wavelengths (as an extreme case) is illustrated in Figure 7 showing that under certain conditions it should be possible to combine beams efficiently with a number of WDM channels. The combination efficiency is expected to exceed $85 \%$ leading to an output power in excess of $85 \mathrm{~W}$. This technique can be scaled to a few number of beams.

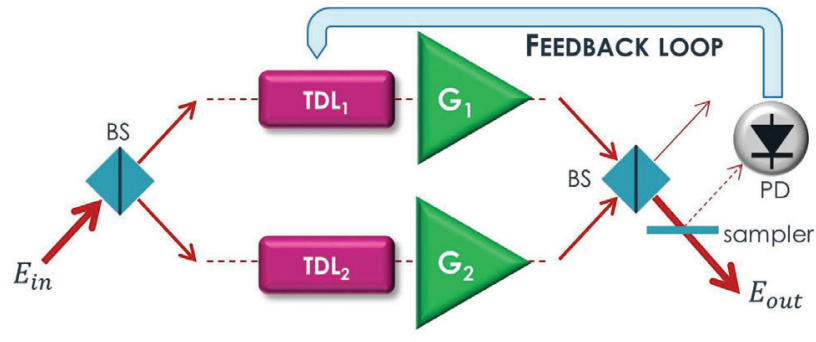

Figure 6. Schematic of the 2 amplifying channels coherent combining setup. BS: 50/50 beamsplitter; G: amplifier gain; PD: photodiode; TDL: tunable delay line.

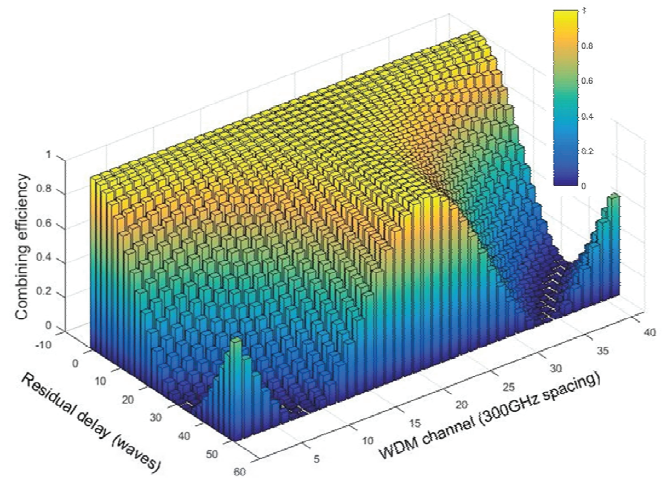

Figure 7. Combining efficiency as a function of the residual delay between 2 amplifying channels, for $40 \mathrm{WDM}$ channels with $300 \mathrm{GHz}$ spacing.

\subsection{High power multiplexing}

The high system capacity can only be achieved by using multiple optical channels, which makes optical multiplexing a critical function. Due to the limited optical power achievable by a single power generation unit, wavelength multiplexing must be performed out after amplification (at least for the final stage before transmission), thus exposing the output stage to high optical power levels. Considering the on-board system, power exceeding $10 \mathrm{~W}$ per multiplexer input is expected. Such a high power optical multiplexer (HP Mux) has been designed by Gooch \& Houseo, using transmission diffraction gratings, according to the principle illustrated in the figure below. A HP Mux breadboard will be realized and tested in the frame of VERTIGO that will exhibit 10 input accesses and will be able to support up to 10 Watts per input access.

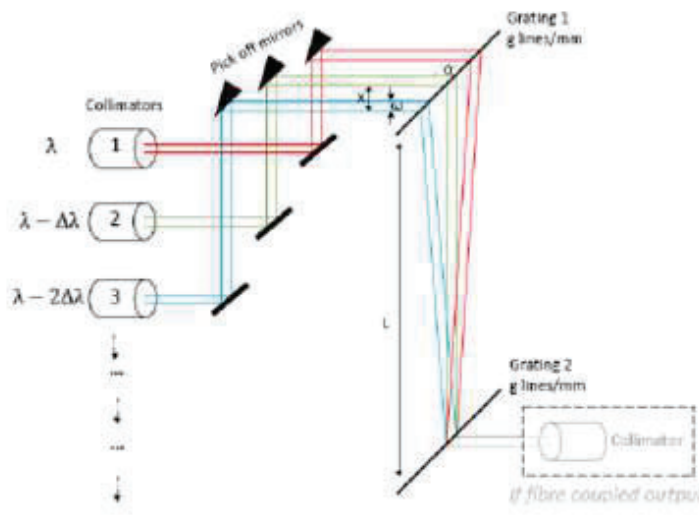

Figure 8. Illustration of the grating based HP Mux. 


\section{OUTDOOR DEMONSTRATION}

\subsection{Demonstration overview}

The purpose of the outdoor demonstration is to assemble most of the key building blocks involved in an optical feeder link and compare different configurations of link (modulation format, optical ground station (OGS) architecture,...) in an environment whose characteristics are comparable to an operational GEO-ground link. To this aim, the outdoor demonstration will implement the same building blocks as the indoor demo previously depicted except the high power sections : optical transmitters and receivers together with coding/interleaving, medium power amplification. The transmitted optical power will be scaled in order to receive signals at a representative power level. The propagation channel will include an optical ground station and a space terminal emulator. The space terminal emulator developed by Thales Alenia Space in Switzerland will allow to emulate the decorrelation between uplink and downlink encountered in a real space-ground link thanks to a structure consisting of two apertures separated by a tunable distance corresponding to the point ahead angle, as depicted in the frame of FEEDELIO demonstration [5].

Two types of ground stations will be used in the frame of this activity:

- A single-aperture telescope equipped with adaptive optics

- A multi-aperture telescope with tip/tilt correction

The Figure 9 hereafter presents a schematic of the overall outdoor demonstration.

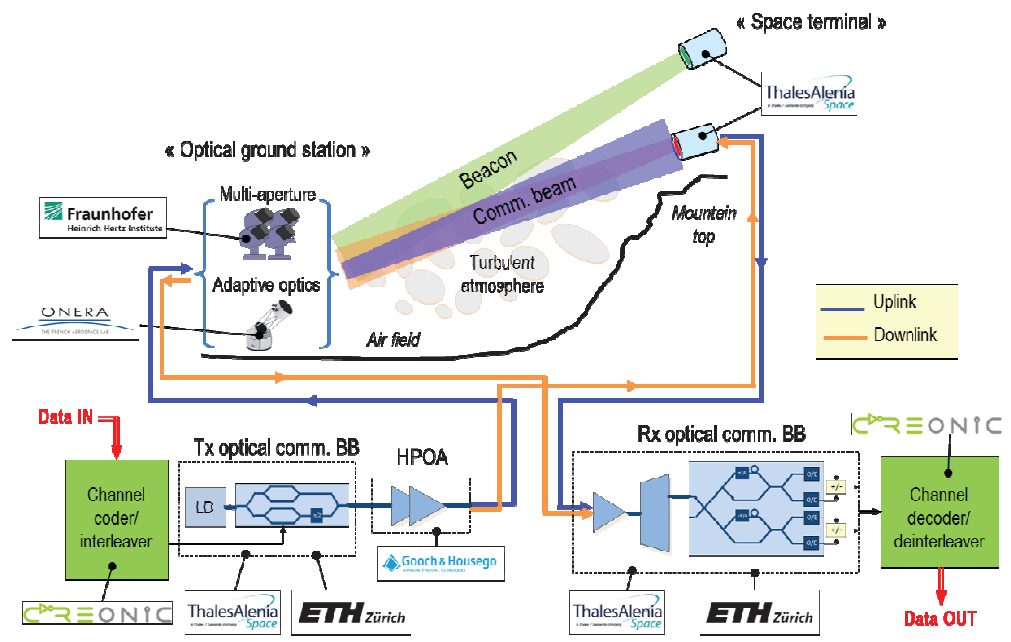

Figure 9. General scheme of the outdoor demonstration.

\subsection{Turbulence conditions}

The outdoor demonstration will be an opportunity to test optical communication in a real case of propagation through a channel disturbed by atmospheric turbulence. This link will be established between the Zimmerwald Observatory of University of Bern and the High Altitude Research Station Jungfraujoch in the Swiss Alps (Figure 10), along a $53 \mathrm{~km}$ slant path. 


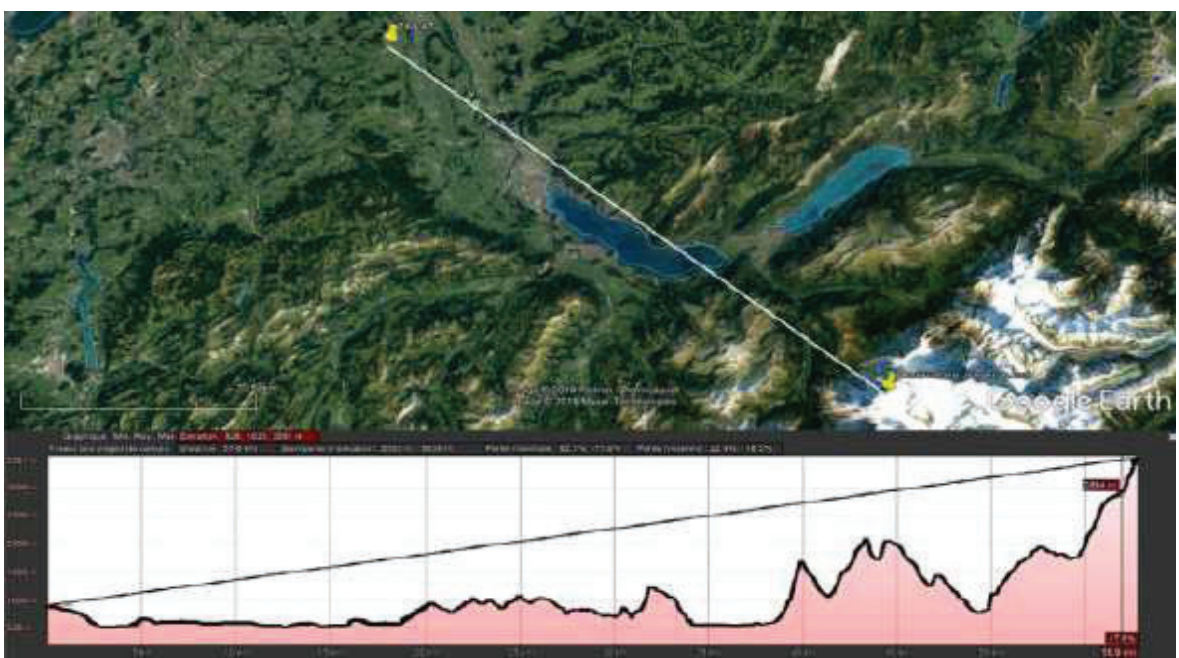

Figure 10. Satellite and topographic view of the Line of Sight.

Propagation conditions fully representative of a real space-ground link cannot be achieved in practice through a groundground link due to different physical parameters all along the line of sight (LoS). Nevertheless it is relevant and useful to perform transmission tests attempting to approach such real-case conditions.

In order to evaluate the representativeness of the considered optical channel, ONERA conducted simulations comparing the statistics of an optical link based on a OGS with adaptive optics used both for downlink correction and for uplink pre-compensation, assuming injection into a single mode optical fiber. Two cases are considered: on one hand a $600 \mathrm{~mm}$ OGS optical channel model based on turbulence conditions assumed to be representative of a real GEO-ground line of sight [5] , and on the other hand a $350 \mathrm{~mm}$ OGS optical channel model based on turbulence conditions estimated from the topography and LoS geometry (Figure10) and accounting for several emulated Point Ahead Angles, alpha. The results, shown in Figure 11 for the uplink (the most challenging), highlight the distribution differences in the two cases. The careful selection of the time of day (or night) for the demo campaign as well as the tuning capability offered in the demo through alpha angle shall be used to approach conditions of a GEO LoS.

\begin{tabular}{|l|c|c|c|c|}
\hline Profile & MOSPAR $50-50 @ 40^{\circ}$ & MOSPAR $90-90 @ 40^{\circ}$ & MOSPAR $50-50 @ 30^{\circ}$ & MOSPAR 90-90@ $30^{\circ}$ \\
\cline { 2 - 5 } $\mathbf{r}_{\mathbf{0}} @ 1.55 \mu \mathrm{m}$ & $9.1 \mathrm{~cm}$ & $4.7 \mathrm{~cm}$ & $7.9 \mathrm{~cm}$ & $4 \mathrm{~cm}$ \\
\hline $\boldsymbol{\theta}_{0} @ 1.55 \mu \mathrm{m}$ & $16.9 \mu \mathrm{rad}$ & $10.2 \mu \mathrm{rad}$ & $11.3 \mu \mathrm{rad}$ & $6.8 \mu \mathrm{rad}$ \\
\hline $\boldsymbol{\sigma}_{\mathrm{x}}^{2} @ 1.55 \mu \mathrm{m}$ & 0.022 & 0.050 & 0.035 & 0.079 \\
\hline
\end{tabular}
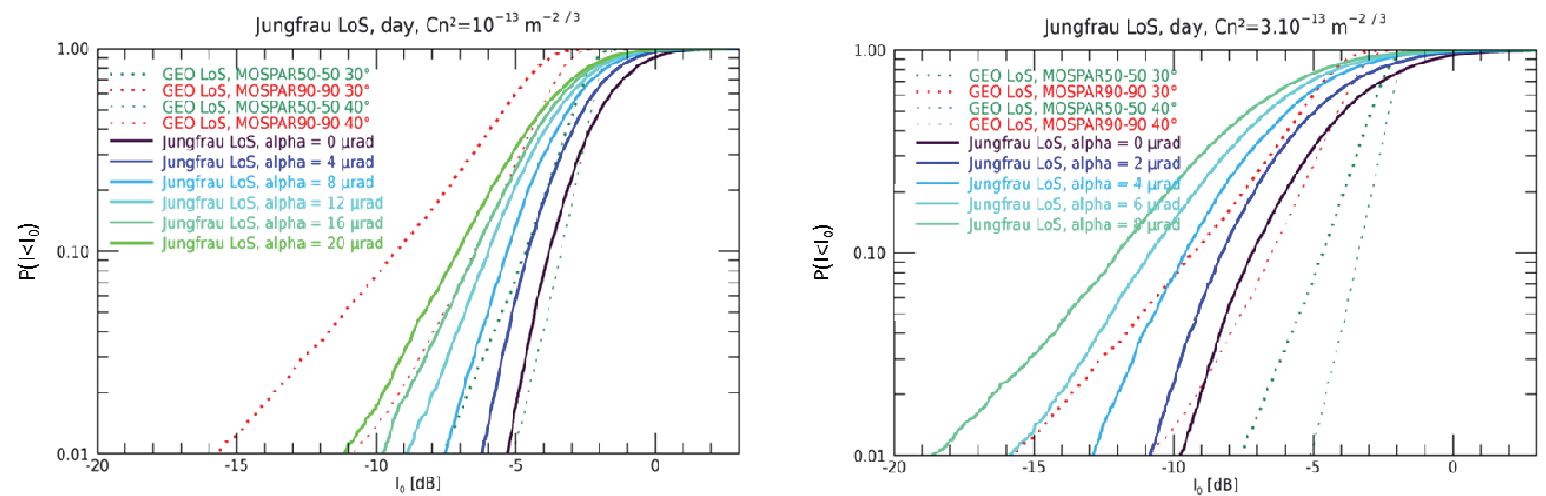

Figure 11. Uplink coupled optical power statistics (cumulated density function) comparison between a GEO LoS with 600 mm OGS and Jungfrau LoS with a 350 mm OGS. 
Besides, during the demonstration, the turbulence conditions will be monitored thanks to adaptive optics data (wavefront sensor measurements, and control commands) so as to facilitate the performance analysis [6].

\subsection{Optical ground stations and space terminal emulator}

As previously stated, two different Optical Ground Station (OGS) set-ups will be deployed on Zimmerwald site: a single aperture OGS making use of adaptive optics and a multi-aperture OGS relying on optical paths diversity for atmospheric effects mitigation. Both OGS will operate in transmission and reception. The single aperture OGS (SA-OGS) is based on GEO feeder link OGS FEELINGS developed by ONERA with an adapted telescope for the VERTIGO ground-ground demo. The station includes a $350 \mathrm{~mm}$ aperture telescope and is equipped with adaptive optics with 15 radial orders of correction for both downlink and uplink (pre-compensation) and tip-tilt management. A partial view of the SA-OGS architecture is presented in Figure 12.

The mutli-aperture OGS (MA-OGS) is provided by Fraunhofer HHI. It relies on an architecture composed of 4 subapertures of $75 \mathrm{~mm}$ diameter and incoherent combining of signals [7]. In this case, scintillation reduction is obtained on the downlink through the averaging over the four sub-apertures (as an alternative to a single larger aperture). The reduction is the most efficient for a distance between the apertures larger than the Fried parameter to ensure a decorrelation between received signals. The signals collected from each aperture are individually photodetected, digitized, allowing to perform delay compensation in the electrical domain, and electronically combining based on the maximum-ratio.

On the uplink, scintillation (including beam wandering) is mitigated through the optical paths diversity and the incoherent optical beam combination in the far-field. Incoherent recombination is based on the use of a separate wavelength per aperture and per signal (data stream) to be transmitted on uplink in order to prevent any interference issue between signals transmitted from the different apertures and to be received on a same detector at receiver side. The combination of the diversity channels on a single detector does not allow coherent transmissions for the uplink. As for the downlink, the distance between the apertures shall be larger than the Fried parameter to achieve a sufficient decorrelation between the optical paths.

As depicted in Figure 12 and 13, the Space Terminal Emulator or Breadboard (STB) provided by Thales Alenia Space in Switzerland will host the different optical communication chains to establish the links with the SA-OGS or the MAOGS. An additional movable aperture in the STB will allow to emulate the Point Ahead Angle (seen from the OGS) between transmit and receive beam.

In addition to the MA-OGS set-up that will be used for the field demonstration in Switzerland, coherent beam recombining at receive side will also be tested as part of a side experiment in Germany using a combination device developed by Thales Research and Technology. The recombination of the signals from the four apertures is performed by a Silicon Photonic Integrated Circuit (Si-PIC) including all necessary functionalities (combiners, delay lines, phase shifters). The schematic of the optical combiner and the corresponding chip are presented in Figure 14. 


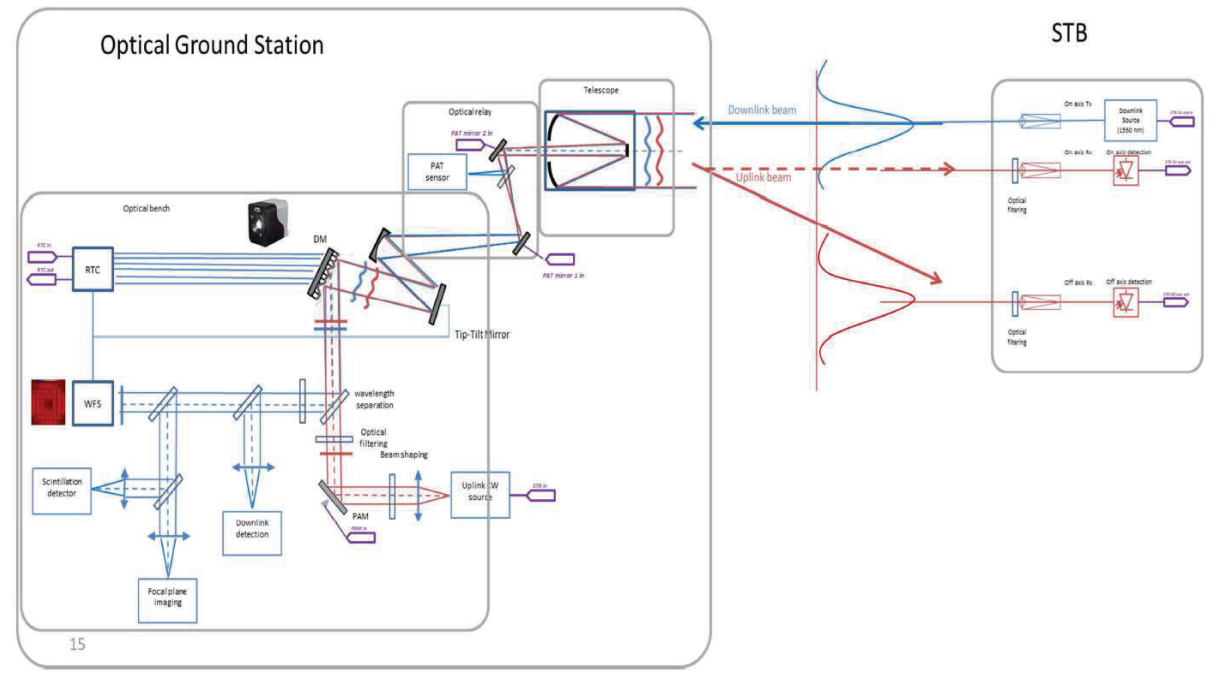

Figure 12. Partial architecture of the SA-OGS developed by ONERA.

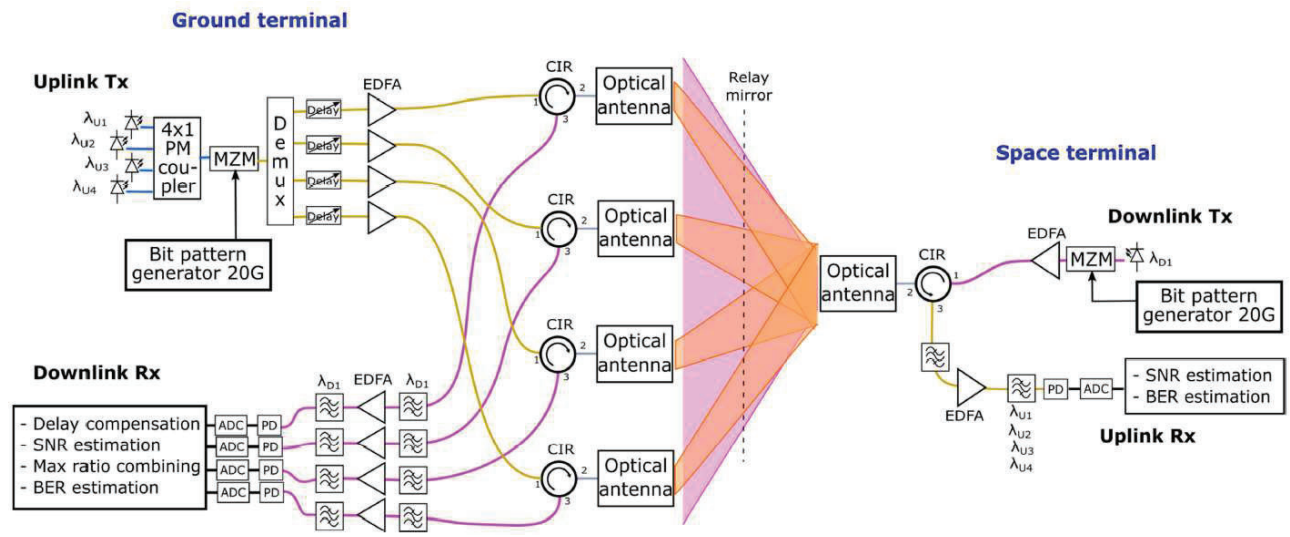

Figure 13. Partial architecture of the MA-OGS developed by Fraunhofer HHI.

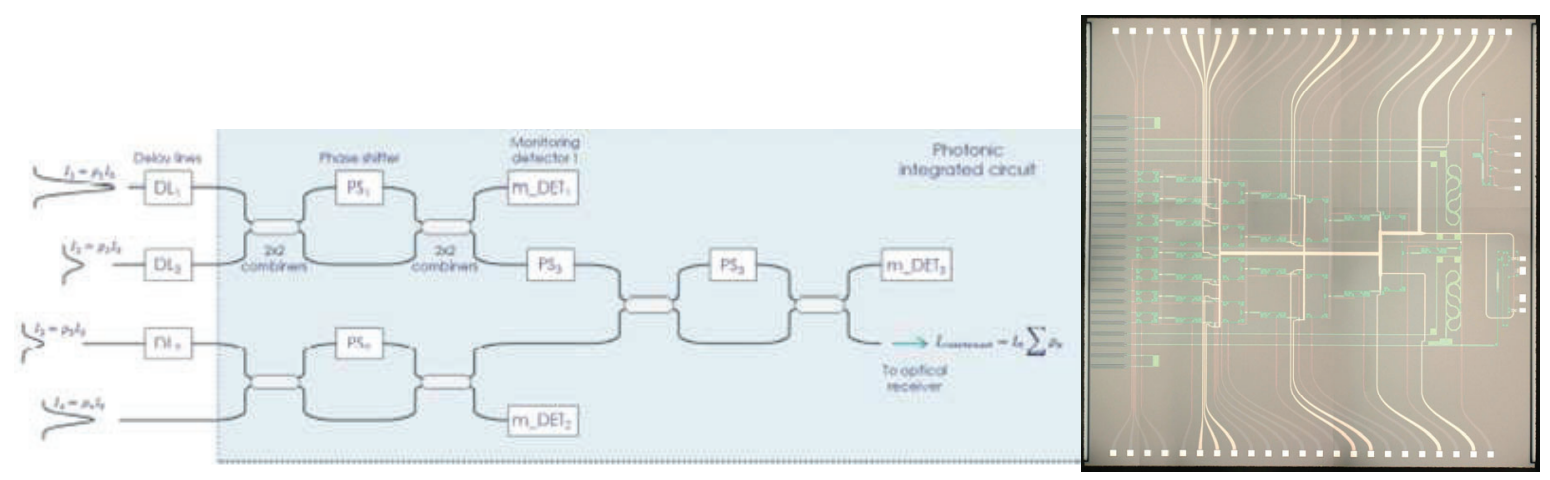

Figure 14. Rx coherent combiner developed by Thales Research and Technology based on a Si-PIC. 


\section{CONCLUSION}

The H2020 VERTIGO project aims at demonstrating the feasibility and assessing the compatibility of a range of key building blocks, techniques and technologies required for the development of high capacity optical feeder links. The project is now progressing well towards the design and implementation of key enablers such as spectral and energy efficient modulation formats and detection schemes, high optical power amplification, high power wavelength multiplexing, coherent optical beam combining at both transmit and receive sides, adaptive optics and multi-aperture optics for mitigation of turbulence effects, as well as digital processing for error correction coding and interleaving. Significant preparation results have already been obtained. The development of representative breadboards is now on going and the perspective of their integration and assessment in lab and outdoor ground-ground demonstrations is shaping up.

The project objectives are very ambitious but it is anticipated that the lessons learnt from such a collaborative project should be decisive and will pave the way to the flight demonstration and operation of future high-throughput satellite systems based on free-space optical links.

\section{ACKNOWLEDGEMENT}

The VERTIGO project has received funding from the European Union's Horizon 2020 research and innovation programme under grant agreement No. 822030.

\section{REFERENCES}

[1] Arapoglou, P.-D., Girault, N., "Optical Feeder Link Architectures for very HTS: Issues and Possibilities," International Conference on Space Optics - ICSO 2018, 2018, Chania, Greece.

[2] Mody, A., Gonzalez, E., "An Operator's View: The Medium-Term Feasibility of an optical Feeder Link for VHTS," IEEE International Conference on Space Optical Systems and Applications - ICSOS 2017, Chania, Greece.

[3] Le Kernec, A., Canuet, L., Maho, A., Sotom, M., Matter, D., Francou, L., "Optical feeder links for high throughput satellites and the H2020 VERTIGO project," Workshop COAT-2019, 2-3 december 2019, ONERA, Châtillon, France.

[4] Project website : http://www.space-vertigo.eu.

[5] Védrenne N., Petit, C., Montmerle-Bonnefois A., Conan, J.-M., Michau, V., Lim C. B., Cassaing F., Meimon, S., Montri, J., "Performance analysis of an adaptive optics based optical feeder link ground station," International Conference on Space Optics (ICSO 2020), 30 March - 2 April 2021, Virtual.

[6] Védrenne N., Montmerle-Bonnefois A., Lim C. B., Petit, C., Sauvage, J.-F., Meimon, S., Perrault, P., Mendez, F., Fleury, B., Montri, J., Conan, J.-M., Michau, V., Sodnik, Z., Voland, C., "First experimental demonstration of adaptive optics pre-compensation for GEO feeder links in a relevant environment," 2019 IEEE International Conference on Space Optical Systems and Applications (ICSOS), Oct 2019, Portland, United States of America, pp. 1-5, doi: 10.1109/ICSOS45490.2019.8978970.

[7] Perlot, N., Hanne, P., Perdigues, J. "Bidirectional 20-Gbit/s OOK link with a 4-telescope array and incoherent signal combining," 2019 IEEE International Conference on Space Optical Systems and Applications (ICSOS), Oct 2019, Portland, United States of America. 